\title{
A high-density genetic map of the Medicago truncatula major freezing tolerance QTL on chromosome 6 reveals colinearity with a QTL related to freezing damage on Pisum sativum linkage group VI
}

\author{
Nadim Tayeh • Nasser Bahrman • Rosemonde Devaux • Aurélie Bluteau • \\ Jean-Marie Prosperi • Bruno Delbreil • Isabelle Lejeune-Hénaut
}

Received: 4 January 2013/ Accepted: 15 April 2013

(C) Springer Science+Business Media Dordrecht 2013

\begin{abstract}
Freezing is one of the most serious abiotic stress factors that affect cool-season legumes. It limits species geographic distribution and causes severe yield losses. Improving tolerance to freezing has long been a main concern for legume breeders. Medicago truncatula Gaertn. has been selected as a model species for legume biology. Various studies have shown significant macrosynteny between $M$. truncatula and agronomically important crop legumes. A major freezing tolerance quantitative trait locus (QTL), herein referred to as Mt-FTQTL6, was previously identified on M. truncatula chromosome 6 . The physical location of this QTL was determined in this study and its corresponding chromosomal interval was enriched with additional markers. Markers were first
\end{abstract}

Electronic supplementary material The online version of this article (doi:10.1007/s11032-013-9869-1) contains supplementary material, which is available to authorized users.

N. Tayeh · N. Bahrman · A. Bluteau · B. Delbreil $(\bowtie)$

Université Lille 1, UMR 1281 SADV, Bâtiment SN2,

59655 Villeneuve d'Ascq Cedex, France

e-mail: bruno.delbreil@univ-lille1.fr

N. Bahrman · R. Devaux · A. Bluteau ·

I. Lejeune-Hénaut

INRA, UMR 1281 SADV, Estrées-Mons BP50136,

80203 Péronne Cedex, France

J.-M. Prosperi

INRA, UMR AGAP, 2 Place Pierre Viala,

34060 Montpellier Cedex, France developed using the draft sequence of $M$. truncatula euchromatin (release versions Mt3.0 and Mt3.5). Because Mt-FTQTL6 was found to coincide with an assembly gap, the Glycine max (L.) Merr. genome sequence was also used to generate markers. Five MtFTQTL6-linked markers were found to be common to a region on Pisum sativum L. linkage group VI harboring a QTL for freezing damage. A subset of markers was tested for transferability across 11 additional legume species. This study lays the groundwork for identifying the molecular basis of MtFTQTL6. Cross-legume markers will be useful in future efforts aiming to investigate the conservation of Mt-FTQTL6 in cool-season legumes and subsequently the existence of common mechanisms for response to freezing between $M$. truncatula and crop legumes.

Keywords Freezing tolerance - Quantitative trait locus (QTL) · Synteny · Medicago truncatula . Glycine max · Pisum sativum

\section{Introduction}

The Fabaceae (Leguminosae) family is the third largest family of flowering plants, comprising more than 700 genera and 20,000 species. It ranks second to Poaceae in terms of economic importance. Most important food and feed legumes belong to the subfamily Papilionoideae. Species that originated from tropical regions are referred to as warm-season legumes; those from 
temperate regions are designated as cool-season legumes (Doyle and Luckow 2003; Gepts et al. 2005).

Due to better moisture availability and longer growing season, fall-sown annual cool-season legumes are superior to spring types in grain or total forage yield (Sadeghipour and Aghaei 2012; Chen et al. 2006; Firıncioğlu et al. 2009). However, harsh winter conditions in predominant production regions greatly limit the use of fall-sown annual crops. Winter stresses also have a crucial impact on the cultivation of perennial cool-season legumes (Bélanger et al. 2006). Improving winter hardiness is expected to expand cultivated areas and increase productivity and yield stability of cool-season legumes.

Winter hardiness of legumes is a complex trait due to its quantitative inheritance and the existence of genotype $\times$ environment interactions (Kahraman et al. 2004; Klimenko et al. 2010; Castonguay et al. 2009). Freezing tolerance has often been used to predict winter hardiness. It is commonly defined as the potential of plants to minimize freezing effects upon a period of exposure to low non-freezing temperatures, known as cold acclimation, during which diverse physiological, molecular and metabolic modifications are induced (Guy 1990). Freezing tolerance is the most determinant factor affecting winter survival across a wide range of environments and genetic evidence of this statement has been provided from different legume species by reporting the co-localization of freezing tolerance quantitative trait loci (QTL) with QTL for winter hardiness (Brouwer et al. 2000; Kahraman et al. 2004; Dumont et al. 2009).

The cool-season legume, M. truncatula, has been chosen as a model molecular-genetic system for legume biology (Cook 1999). A draft sequence of its euchromatin, comprising approximately $94 \%$ of all genes, is currently available (assembly version Mt3.5; Young et al. 2011). Three QTL for freezing tolerance at the vegetative stage were identified in $M$. truncatula after a cold acclimation period using the F83005$5 \times$ DZA045-5-derived population (LR3 population; Komlan et al. 2013). They were mapped on chromosomes 1, 4 and 6. The major QTL on chromosome 6 (Mt-FTQTL6) accounted for $40 \%$ of the freezing tolerance variation. The simple sequence repeat (SSR) marker MTIC153, derived from an expressed sequence tag (EST) with a Bcl-2-associated athanogene annotation (Julier et al. 2003), was reported as the peak marker for this QTL but its sequence could not be located on the M. truncatula chromosome 6 pseudomolecule. In parallel, a QTL related to freezing damage was detected on $P$. sativum linkage group (LG) VI under both field (WFD 6.1; Lejeune-Hénaut et al. 2008) and controlled conditions (FD164.c; Dumont et al. 2009) using the Champagne $\times$ Teresederived population (Pop2 population). M. truncatula chromosome 6 and $P$. sativum LG VI were reported to share conserved macrosynteny (Aubert et al. 2006; Bordat et al. 2011). However, no common molecular markers were available to determine whether MtFTQTL6 and WFD 6.1/FD164.c are located on corresponding genomic regions.

"The objectives of the present study were to: (1) place Mt-FTQTL6 on the M. truncatula genome sequence; (2) enrich the QTL interval with markers; (3) compare the chromosomal location of Mt-FTQTL6 with that of $P$. sativum WFD 6.1/FD164.c; and (4) provide cross-species markers that would permit the examination of Mt-FTQTL6 conservation in other legume species.

\section{Materials and methods}

Marker development

\section{Bacterial artificial chromosome (BAC)-derived markers}

Two markers from the integrated genetic map of $M$. truncatula chromosome 6 (University of Minnesota 2006; http://www.medicago.org/genome/map.php), 004F08 and h2-166b10a, were transferred to the LR3 genetic map. These markers mapped on both sides of marker MTIC153 (Komlan et al. 2013). The 21 BAC clones spanning the interval between 004F08 and h2166b10a on $M$. truncatula genome assembly version Mt3.0 were used to generate primer pairs for candidate BAC-derived markers. With the release of the Mt3.5 genome assembly version and thus updates of $\mathrm{BAC} /$ contig order and orientation, the interval was extended to include 11 additional BAC clones located down to mth2-9p17 [National Center for Biotechnology Information (NCBI) GenBank accession (GB acc.) AC146307]. Coding sequences from target BAC clones were submitted to BLAST (Basic Local Alignment Search Tool; Altschul et al. 1990) searches against the NCBI $M$. truncatula nucleotide and high-throughput 
genomic sequence (HTGS) databases to avoid redundancy. Selected non-redundant sequences were searched for highly similar EST/messenger RNA from other legume species. Homologous sequences were aligned using MultAlin (Corpet 1988). Primers were designed on conserved exonic, preferentially intron-spanning, regions. When no primers for candidate gene-based markers could be obtained, markers were developed on non-repetitive intergenic microsatellite-flanking sequences. Microsatellite motifs were identified using Perfect Microsatellite Repeat Finder (http://sgdp.iop. kcl.ac.uk/nikammar/repeatfinder.html). The minimum number of repeats was set to 5 .

\section{Synteny-based markers}

MTIC153-corresponding EST (GB acc. AL369679) and coding sequences from BAC clones encompassing the boundaries of Mt-FTQTL6 were used as BLAST queries to identify similar sequences in the G. max genome. Candidate regions syntenic to Mt-FTQTL6 could thus be located on G. max chromosomes 5, 9, 10, 16, 17 and 20. In M. truncatula, homoeologous regions to Mt-FTQTL6 could be located on chromosomes 1 and 5 following $M$. truncatula-M. truncatula and M. truncatula-G. max BLAST and reverse BLAST searches. Among all blocks syntenic to Mt-FTQTL6 in G. max and M. truncatula, genes from the Mt-FTQTL6-containing region exhibited the highest similarity to those on G. max chromosomes 9 and 16. Both G. max regions are homoeologous and derived from the G. max lineage-specific paleotetraploidization (ca. 13 million years ago; Schmutz et al. 2010). Protein sequences from target $G$. $\max$ regions were used to search for putative homologous M. truncatula EST, HTGS, or nucleotide sequences using TBLASTN. NCBI legume EST databases and the Lotus japonicus (Regel) K. Larsen genome sequence (http://www.kazusa.or. jp/lotus/blast.html) were also regularly searched for highly similar counterparts ( $>85 \%$ sequence identity). All homologous sequences obtained by BLAST searches were translated in silico (ExPASy Translate Tool; http:// web.expasy.org/translate), aligned and used for phylogenetic tree construction (phylogeny.fr; Dereeper et al. 2008). Orthologous sequences to those from G. $\max$ chromosomes 9 and/or 16 that were identified by the phylogenetic method were retained for primer design.

In all cases, primers were designed using the Primer3 tool (Rozen and Skaletsky 2000) with default parameters. Maximum product length was generally limited to
$500 \mathrm{bp}$. Degenerate nucleotides were included in some primer sequences to ensure cross-species amplification. The PrimerSearch tool, available at http://www.legoo.org , was used to check the specificity of the generated primer pairs for PCR amplification in M. truncatula.

Plant material

Markers developed in this study were first scored on recombinant inbred lines (RIL) from $M$. truncatula LR3 and $P$. sativum Pop2 populations. The LR3 population (178 F2:5 RIL) was developed by singleseed descent from a cross between the French freezingtolerant line F83005-5 and the Algerian freezingsensitive line DZA045-5 (INRA, UMR AGAP, Montpellier, France; http://www1.montpellier.inra.fr/BRCMTR). This population was used to map QTL for freezing tolerance under controlled conditions (Komlan et al. 2013). Total genomic DNA of the 178 RIL from the LR3 population was provided by Dr. MarieLaure Pilet-Nayel (INRA, UMR IGEPP, Rennes, France). The Pop2 population (164 F2:8 RIL) was derived from a cross between $P$. sativum lines Champagne and Terese (Loridon et al. 2005). QTL related to freezing damage were identified in this population both under field and controlled conditions (Lejeune-Hénaut et al. 2008; Dumont et al. 2009).

Large M. truncatula and $P$. sativum populations were used to determine the order of co-segregating markers on LR3 and Pop2 linkage maps, respectively. A population comprising $454 \mathrm{M}$. truncatula F6 plants was produced from the self-pollination of two residual heterozygous lines (RHL): 76-06 (237 plants) and 76-11 (217 plants). The parental lines 76-06 and 76-11 are sisters of LR3 RIL\#76. All three have residual heterozygosity in the chromosomal segment harboring Mt-FTQTL6 and are largely homozygous throughout the rest of the genome (based on genotype data from 3-6 evenly-spaced microsatellite markers per chromosome). A P. sativum population of 1,438 individuals was obtained from the self-pollination of 132 parental plants heterozygous for WFD 6.1/FD164.c. These latter were selected among 189 total plants obtained from crosses between line Eden and eight progenies of a two-backcross plant that are heterozygous at WFD 6.1/FD164.c. The two-backcross plant was derived from a cross between Eden (female, recurrent parent) and Pop2 RIL\#38 (male, donor parent). RIL\#38 carries alleles from Champagne at WFD 6.1/FD164.c. Large 
populations were grown in the greenhouse at an average temperature of $18 / 14{ }^{\circ} \mathrm{C}$ (day/night) and a 16/8-h light/dark photoperiod.

Eleven legume species belonging to the hologalegina, phaseoloid/millettioid and genistoid clades of the Papilionoideae subfamily (Doyle and Luckow 2003) were used for marker transferability tests (Online Resource 1), with one or two cultivars/accessions per species. Seeds were spread on wet filter papers contained in Petri dishes and left to germinate at room temperature in the dark.

Leaflets from the $M$. truncatula RHL-derived population and entire seedlings from the set of eleven legume species were collected on 1-month-old and 7-10-day-old plants, respectively, frozen in liquid nitrogen and stored at $-80{ }^{\circ} \mathrm{C}$ until DNA extraction. Stipules from 1-month-old $P$. sativum plants were lyophilized and kept at $-20{ }^{\circ} \mathrm{C}$.

DNA isolation, PCR amplification and PCR product sequencing

Total genomic DNA extraction was performed using a Nucleospin 96 Plant II kit (Macherey Nagel, Düren, Germany) according to the manufacturer's instructions. Buffer PL1 was used for cell lysis. Elutions were performed with $2 \times 100 \mu \mathrm{L}$ of pre-warmed ultrapure water $\left(70^{\circ} \mathrm{C}\right)$. DNA quality and quantity were assessed by agarose gel electrophoresis.

PCR amplification and sequencing were carried out as described in Online Resource 2. Briefly, PCR reactions were performed in a final volume of $15 \mu \mathrm{L}$ with $10 \mathrm{ng}$ of genomic DNA template. Two different thermal cycling protocols were employed and are referred to as touchdown or three-step protocol. PCR products were sequenced using BigDye Terminator v3.1 chemistry (Applied Biosystems, Foster City, California, USA) on a 3130xl Genetic Analyzer (Hitachi/Applied Biosystems). $M$. truncatula and $P$. sativum marker sequences are provided in FASTA format in Online Resource 12.

Polymorphism identification and genotyping techniques

Polymorphism tests were run using $8-16$ individuals including parental lines and segregating progeny. Lines with residual heterozygosity in chromosomal segments containing Mt-FTQTL6 and WFD 6.1/FD164.c were particularly valuable as they brought direct evidence for the linkage of markers to target regions. SSR and insertion/deletion (indel) polymorphisms were detected and genotyped using either agarose or capillary gel electrophoresis, depending on parental allele sizes. Detection of single nucleotide polymorphisms (SNP) was performed using a high-resolution melting technique (HRM). Genotyping of SNP markers was conducted either by HRM or competitive allele-specific PCR chemistry (KASPar; KBiosciences, Hoddesdon, UK) for largerscale genotyping. Allele-specific and common primers for markers genotyped by KASPar are provided in Online Resource 8. Additional method details are given in Online Resource 2.

\section{Linkage analysis and QTL mapping}

The genetic linkage map of the LR3 population was described in Komlan et al. (2013) and that of the Pop2 population was described in Loridon et al. (2005) and Aubert et al. (2006). The framework map of $M$. truncatula chromosome 6 , used in this study, comprises four SSR, five random amplified polymorphic DNA (RAPD) and five amplified fragment length polymorphism (AFLP) markers (Online Resource 3a). Markers genotyped in the $P$. sativum Pop2 population were mapped on an 18-loci framework map of LG VI [nine SSR, four RAPD, one AFLP and three gene-based markers] (Loridon et al. 2005; Aubert et al. 2006). Markers having names starting with "NT" together with $P$. sativum markers MTIC153 and Ps92K09T were developed in this study. Marker Cabb was previously used by Bordat et al. (2011) to genotype multiple $P$. sativum segregating populations. A new primer set for Cabb was used in this study: forward primer 5'-TGTTCATTTGTTGTGTTTGAAGC- ${ }^{\prime}$ and reverse primer 5'-AAATTATAGCTTTTGCCTTAACACG$3^{\prime}$. Full information regarding genetic distances between different $M$. truncatula markers is given in Online Resource 3. As described above, large M. truncatula and $P$. sativum segregating populations were derived from parental lines showing heterozygosity at Mt-FTQTL6 and WFD 6.1/FD164.c, respectively. They were thus treated as F2 populations and used to construct high-resolution linkage maps for target regions. In all mapping analyses, the goodness-of-fit to expected 
segregation ratios, $1: 1$ or $1: 2: 1$ accordingly, were assessed by the Chi squared test implemented in JoinMap 4.1 software (Van Ooijen 2006). Similar to MTIC153, BAC-derived and synteny-based markers developed in this study all exhibited distortion from the expected 1:1 segregation ratio (significant at $P<0.01$ ) within the LR3 population showing a higher DZA045-5 allele frequency. No segregation distortion was noted for new markers in the other mapping populations. The grouping of markers into LG was evaluated using independent logarithm of odds (LOD). The map order was estimated using JoinMap 4.1 with the regression mapping algorithm (RM; default parameters: recombination frequency $<0.4, \mathrm{LOD}>2$ ) and the Kosambi mapping function (Kosambi 1944). For comparison, maximum likelihood mapping (MLM) was also used. In all cases, the marker order was the same with both algorithms. Linkage groups were drawn using MapChart software 2.2 (Voorrips 2002).

QTL mapping analyses were performed using interval mapping implemented in MapQTL 6 software (Van Ooijen 2009). Freezing tolerance data, scored on the LR3 population by Komlan et al. (2013), were considered. For the Pop2 population, phenotypic data obtained under field experiments (Lejeune-Hénaut et al. 2008) and those from controlled conditions (Dumont et al. 2009) yielded the same QTL position. Only the LOD profile for data scored under controlled conditions is shown in this paper. LOD thresholds for QTL detection on M. truncatula chromosome 6 and $P$. sativum $\mathrm{LG}$ VI were estimated using permutation tests with a $P$ value of 0.05 and 10,000 permutations.

\section{Results}

Location of Mt-FTQTL6 on M. truncatula

chromosome 6 pseudomolecule

Twenty-five primer pairs for candidate gene-based (SNP/indel; 21 primer pairs) and intergenic SSRflanking (four primer pairs) markers were designed based on full sequences of $16 \mathrm{M}$. truncatula BAC clones from eight different contigs (see Materials and Methods). Seventeen markers originating from 14 different BAC clones were found to be polymorphic between F83005-5 and DZA045-5 (Online Resource 4). All BAC-derived markers mapped close to marker MTIC153 in an 8.3-centiMorgan (cM) interval
(Online Resource 3b). The resulting genetic order is not completely concordant with the position of related BAC clones either in $M$. truncatula assembly version Mt3.0 or Mt3.5 (Online Resource 5). It agrees more closely with the BAC contig order/orientation proposed by version Mt3.5 for the upper part of the region containing Mt-FTQTL6; however, it agrees with the Mt3.0 version in the lower part. The orientation of BAC contigs could not be resolved due to the absence of recombination events. Interval mapping confirmed previous results regarding Mt-FTQTL6 position with marker MTIC153 as the closest marker to its peak. The $95 \%$ confidence interval of Mt-FTQTL6 was located between markers NT6001 and NT6019, and thus between BAC clones mth2-156d20 (GB acc. AC157779) and mth2-48m20 (GB acc. AC225497) [Online Resources 5 and 6].

Construction of a gene-based linkage map for Mt-FTQTL6 using synteny between

M. truncatula and G. max

The Mt-FTQTL6 confidence interval, bracketed by markers NT6001 and NT6019, coincides with an assembly gap on the M. truncatula chromosome 6 pseudomolecule (Online Resource 5). A syntenybased approach using G. max as a reference genome was carried out in order to develop close markers to Mt-FTQTL6. Candidate orthologs for genes underlying Mt-FTQTL6 were assumed to exist among genes contained in an approximately $867-\mathrm{kb}$ genomic region on G. max chromosome $16 \quad(35.17-36.04 \mathrm{Mb})$ between Glyma16g31930 and Glymal6g32950 and/ or in its homoeologous region on chromosome 9 (Glyma09g26080-Glyma09g28090; 32.33-35.09 Mb). Glyma16g31930 and Glymal6g32950 have their M. truncatula counterparts directly adjacent to gene/ BAC sequences corresponding to markers NT6001 and NT6019. Primer pairs corresponding to 17 distinct genes yielded successful amplification from F83005-5 and DZA045-5 while primers corresponding to five other genes did not produce any amplification (Online Resource 7). Twelve markers were found to be polymorphic between F83005-5 and DZA045-5 and mapped within a 2.1-cM interval around MTIC153 (Online Resource 3c). Six BAC clones could be identified as having potentially originated from the Mt-FTQTL6-containing region based on markers located within or in contiguous clones. These include 
two distinct unanchored BAC clones [mth2-154j21 (GB acc. AC146807) and mth2-53124 (GB acc. AC229695)] and four others forming a single contig that was formerly placed nearer the distal end of $M$. truncatula chromosome 6 (clone name, GB acc.: mth2-12e18, AC229727; mth2-1515, AC130804; mth2-172p22, AC146818; mth2-50m10, AC174372).

Synteny between Mt-FTQTL6 and P. sativum WFD 6.1/FD164.c

Twenty-nine BAC-derived and synteny-based markers originating from 26 distinct genes yielded positive amplification in $P$. sativum lines, Champagne and Terese, nine of which were polymorphic (Online Resources 4 and 7). Seven markers were mapped in the WFD 6.1/FD164.c region while two others, viz. BACderived markers NT6039bis and NT6044, mapped approximately $40 \mathrm{cM}$ apart at the top of $P$. sativum LG VI (Fig. 1b). Five anchor markers were obtained between Mt-FTQTL6 and WFD 6.1/FD164.c corresponding genomic regions, revealing QTL synteny (Fig. 1). MTIC153 and co-segregating markers NT6067, Ps92K09T and NT6092Ps are located at $0.3 \mathrm{cM}$ from markers NT6012, NT6011 and Cabb, and at $2.6 \mathrm{cM}$ from marker NT6032 (Fig. 1b).

In order to develop additional markers in the upper part of WFD 6.1/FD164.c, new primer pairs were designed in conserved genes between the upper region of Mt-FTQTL6 and corresponding G. max regions (Online Resource 7). Out of nine markers with positive amplification from Champagne and Terese, NT6083 and NT6078 were found to be polymorphic and to cosegregate with other synteny-based markers (Fig. 1b).

Colinearity between $M$. truncatula, $P$. sativum and G. max

To enable high-resolution comparative analyses, markers linked to $M$. truncatula and $P$. sativum syntenic freezing tolerance QTL-containing intervals were used to screen large segregating populations, comprising 454 and 1,438 plants, respectively. The marker order on $M$. truncatula and $P$. sativum LGs was found to be almost identical to that of the corresponding genes on G. max chromosome 16, indicating a high level of colinearity between the three species (Fig. 2). A unique local rearrangement in marker/gene order was observed between $P$. sativum and $G$. max. This concerned markers NT6078 and NT6083 (Online Resource 9). Data concerning the comparison of $M$. truncatula and $P$. sativum LGs with the orthologous region on G. max chromosome 9 are provided in Online Resource 9.

Cross-legume transferability of synteny-based markers

In addition to $M$. truncatula and $P$. sativum, the transferability of 27 synteny-based markers was evaluated across 11 legume species (Online Resource 1). Online Resource 10 summarizes the results of PCR amplification. Seventeen to 24 markers (63-89\% of the total tested markers) yielded positive amplification from each cultivar/accession. Thirteen markers were transferable across all tested species, among which eight have been shown above to be located close to Mt-FTQTL6 and/or WFD 6.1/FD164.c. In contrast to markers targeting exons, intron-spanning markers exhibited differences in product sizes across species with seven out of 16 markers showing size variations exceeding 100 bp (Online Resources 10 and 11).

\section{Discussion}

Synteny as a potential tool for circumventing difficulties in M. truncatula genome assembly

With the advent of sequencing technologies, large genomic resources are being developed from a wide variety of plant species including legumes. Besides genome sequencing and assembly efforts in $M$. truncatula, the gene-rich genomic regions of the model legume L. japonicus (Sato et al. 2008) and the crop legume Vigna unguiculata (L.) Walp (Timko et al. 2008) together with the whole genomes of the economically important warm-season crop legumes G. $\max$ (Schmutz et al. 2010) and Cajanus cajan L. (Varshney et al. 2012) have been sequenced and assembled. Since Mt-FTQTL6 was found to coincide with an assembly gap, it was thus possible to turn to other sequenced legume species for marker development. The gene-space sequence from $L$. japonicus and whole-genome sequence from G. max were considered here. Macro- and microsynteny have already been reported between $M$. truncatula and L. japonicus (Choi et al. 2004a; Cannon et al. 2006; Young et al. 


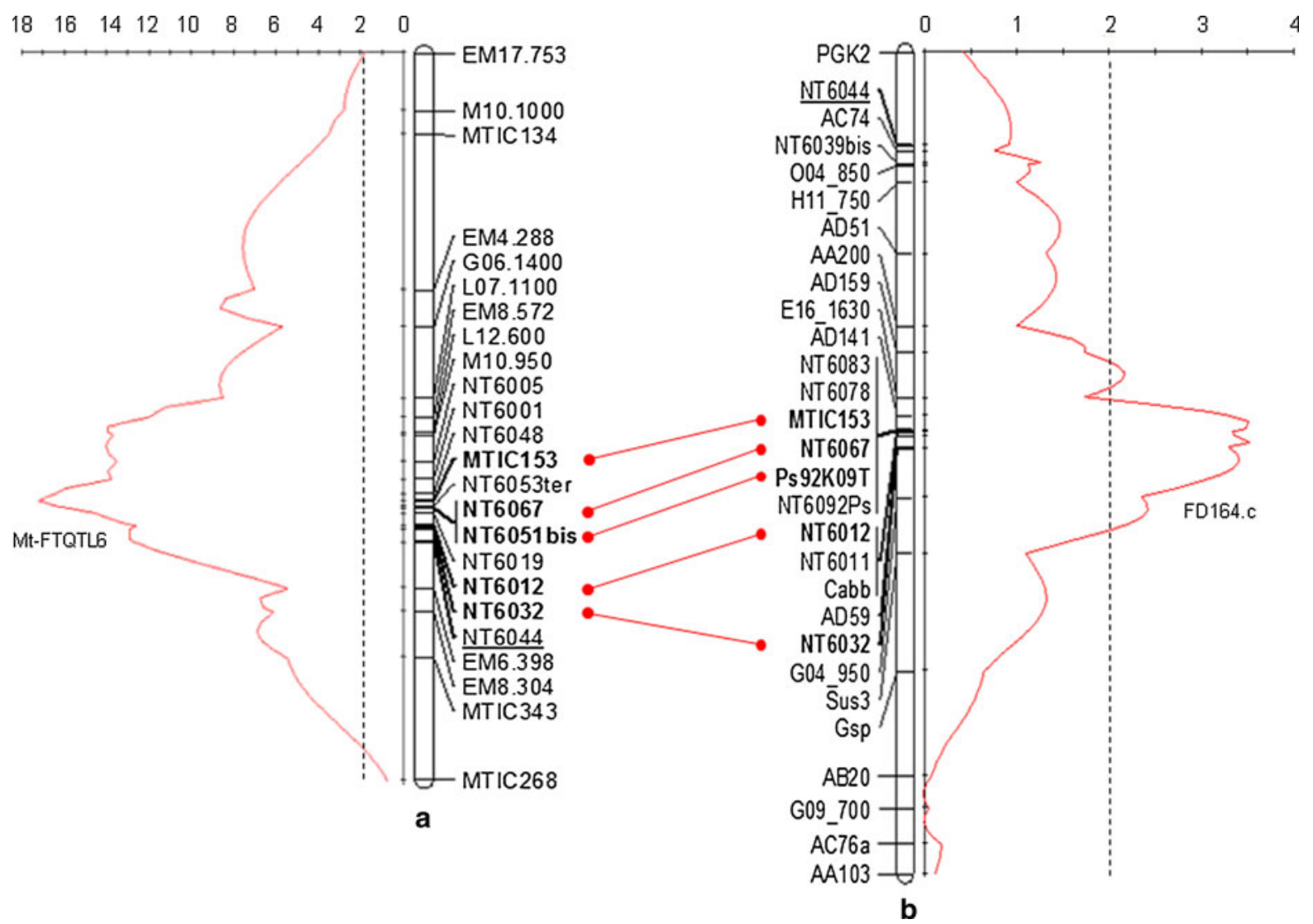

Fig. 1 Anchor markers between M. truncatula and P. sativum genomic regions harboring freezing tolerance-related QTL: Mt-FTQTL6 on M. truncatula chromosome 6 (a) and FD164.c on $P$. sativum linkage group VI (b). Solid red lines link anchor markers (in bold) between QTL-containing regions. The $M$. truncatula and P. sativum common marker, NT6044, is underlined on both linkage groups. It mapped outside the FD164.c confidence interval $(41.3 \mathrm{cM}$ distant from marker NT6083 or other coincident markers) to the top of $P$. sativum linkage group VI. For the sake of clarity, some of the tightly

2011) as well as between M. truncatula and G. $\max$ (Choi et al. 2004a; Mudge et al. 2005; Schlueter et al. 2008; Shin et al. 2008; Joseph et al. 2009; Young et al. 2011). The most similar genes to those from the target region on $M$. truncatula chromosome 6 were identified on L. japonicus chromosome 2 and G. max chromosomes 9 and 16. Because the assembly of the MtFTQTL6 putative orthologous region in L. japonicus is not complete, marker development was conducted using only G. $\max$ as a reference genome. High conservation of gene content and order between $M$. truncatula and G. max was observed based on the number of Mt-FTQTL6-linked markers that could be linked or co-segregating markers on the M. truncatula chromosome 6 were excluded from the figure (see Online Resource 3 for full information). Horizontal axes show LOD values. Dashed black lines correspond to the significance thresholds estimated by permutation tests (LOD $=1.8$ for $M$. truncatula chromosome 6 and LOD $=2$ for $P$. sativum linkage group VI). Maximum LOD scores for Mt-FTQTL6 $(\mathrm{LOD}=17.21)$ and FD164.c $(\mathrm{LOD}=3.45)$ were obtained for both MTIC153 and co-segregating markers

obtained and their order on the genetic linkage maps constructed using either the LR3 or the RHL-derived population. These results extend previous observations on synteny conservation between $M$. truncatula and $G$. max and are particularly important since they concern $M$. truncatula chromosome 6, reported to be the shortest among all eight $M$. truncatula chromosomes, the most heterochromatic (Kulikova et al. 2001) and the richest in nucleotide-binding siteleucine-rich repeat genes (Zhu et al. 2002). Taking into account the effectiveness of this strategy, it can be argued that similar efforts may be helpful to provide markers linked to other residual gaps in the $M$. 


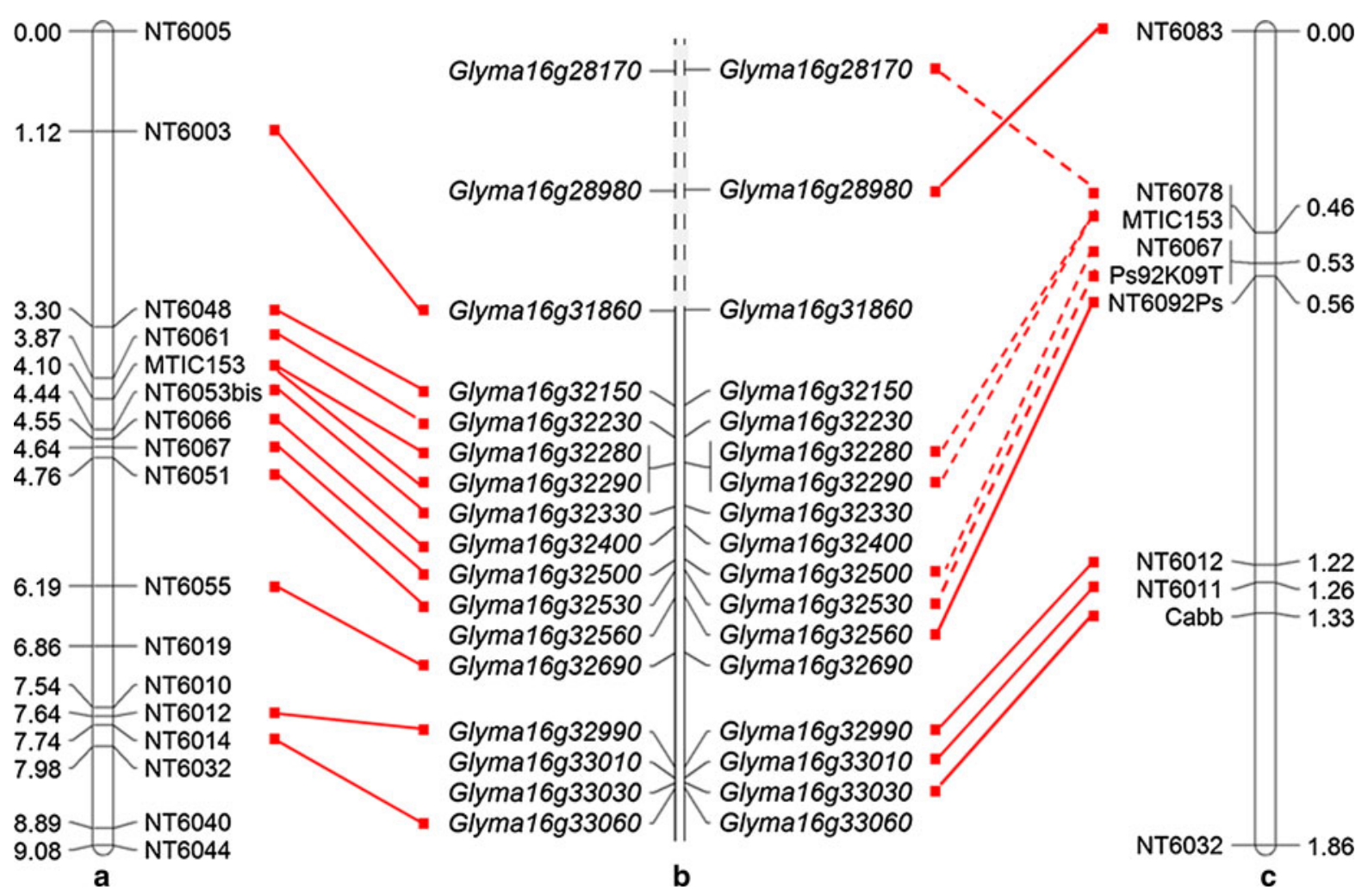

Fig. 2 Comparative linkage groups for M. truncatula (a), G. $\max (\mathbf{b})$ and $P$. sativum (c) showing conservation of marker/gene order on orthologous chromosomal segments. The G. maxcorresponding linkage group represents the gene order on $G$. max chromosome 16 according to the genome assembly version 1.01. It was constructed using physical distances between different genes with $250 \mathrm{~kb}$ being equivalent to one scale unit. For presentation clarity, exceptions to gene spacing were made

truncatula genome and, as shown in this study, may help assign unanchored BAC clones to their corresponding genomic regions. The current $M$. truncatula genome assembly version (Mt3.5) still comprises 273 physical gaps and 146 unanchored BAC/BAC pools (Young et al. 2011).

\section{Conservation of Mt-FTQTL6 in crop legumes}

The conservation of genomic regions responsible for quantitative traits has been reported between different plant species and for multiple traits comprising, for instance, QTL for resistance to Pyricularia grisea between Hordeum vulgare L. and Oryza sativa L. (Chen et al. 2003), bud burst QTL in Quercus robur L. and Castanea sativa Mill. (Casasoli et al. 2006) and fruit quality QTL among Fragaria $\times$ ananassa and for Glyma16g28170 and Glyma16g28980 (dotted bar). Glyma16g28170 and Glyma16g28980 are located at 3.01 and $2.23 \mathrm{Mb}$ from Glyma16g31860, respectively. Solid red lines link markers from $M$. truncatula and $P$. sativum linkage groups to corresponding G. max genes. Dotted red lines are used in the case of co-segregating markers (where no certainty for order conservation with $G$. $\max$ genes exists at present)

Malus or Prunus populations (Zorrilla-Fontanesi et al. 2011). It is often assumed that genes with conserved functions may underlie syntenic QTL. Colinearity between chromosomal regions harboring grain protein content QTL in H. vulgare and Triticum turgidum L. ssp. dicoccoides suggested orthologous NAC transcription factors as responsible for the trait variation in both species (Distelfeld et al. 2008). In legumes, syntenic QTL have been identified among different species. In fact, one of the first evidences for plant orthologous QTL was provided by Fatokun et al. (1992) who reported that conserved regions of Vigna radiata (L.) Wilczek and $V$. unguiculata genomes contain seed weight QTL. These QTL from $V$. radiata and/or $V$. unguiculata were later reported to be conserved in P. sativum (Timmerman-Vaughan et al. 1996), G. max (Maughan et al. 1996), Cicer arietinum 
L. and L. japonicus (Gondo et al. 2007). A genomic region responsible for the variation in days to flowering was suggested to be conserved in six legume species including Vicia faba L., L. japonicus, $P$. sativum, Lupinus angustifolius L., C. arietinum and $M$. truncatula, based on synteny between corresponding chromosomes (Cruz-Izquierdo et al. 2012). Almost at the same time, D'Erfurth et al. (2012) have shown that syntenic chromosomal regions containing seed weight QTL on M. truncatula chromosome 5 and $P$. sativum LG I harbor orthologous subtilisin-like proteases responsible for the QTL effects.

The present study provided the first evidence for Mt-FTQTL6 conservation in P. sativum. Colinearity between the genomic regions containing Mt-FTQTL6 and $P$. sativum WFD 6.1/FD164.c (Lejeune-Hénaut et al. 2008; Dumont et al. 2009) was determined based on five common gene-based markers. Attempts to compare Mt-FTQTL6's position with that of QTL for winter hardiness and/or freezing tolerance from Trifolium pratense L. (Klimenko et al. 2010), Lens culinaris Medik. (Kahraman et al. 2004) and V. faba (Arbaoui et al. 2008) were not successful. This is mostly due to the genomic markers used for linkage map construction in the respective studies not being suitable for comparative mapping. A survey of the literature yielded potentially important data from a study on Medicago sativa L. subsp. sativa. Castonguay et al. (2010) have reported that a sequencerelated amplified polymorphism marker whose sequence matches $M$. truncatula BAC clone mth29p17 (GB acc. AC146307) is associated with freezing tolerance in recurrently selected populations of $M$. sativa subsp. sativa improved for their response to freezing. The fact that marker NT6016 developed from the mth2-9p17 sequence immediately flanks the Mt-FTQTL6 confidence interval (Online Resource 3bc) and that the genomes of M. truncatula and M. sativa are highly syntenic (Julier et al. 2003; Choi et al. 2004b) make Castonguay et al.'s finding potentially important in the context of the present work.

Cross-legume markers (Online Resource 10) are useful to: (1) investigate whether the aforementioned freezing tolerance loci from $M$. truncatula and $M$. sativa subsp. sativa reside in corresponding genomic regions; (2) further examine Mt-FTQTL6 conservation in mapping populations and germplasm collections of agronomically important cool-season legumes by studying the association of markers with phenotypic variation for freezing tolerance; and (3) assist the assembly of genomic contigs originating from Ft-MtQTL6 orthologous regions in different sequenced legume species allowing comparative genomic sequence analysis. In addition to syntenybased markers, some of the BAC-derived markers would also be successfully transferable across legumes and similarly used for the above-mentioned purposes.

Finally, in the light of the above results and discussion, it appears that one of the primary goals of future molecular studies on legume freezing tolerance should be to investigate the orthology of the genes underlying Mt-FTQTL6 and syntenic loci from different species. In line with this, and as a first step forward, fine mapping and map-based cloning of Mt-FTQTL6 are currently ongoing taking advantage of the markers reported here.

Acknowledgments The authors wish to thank Drs. MarieChristine Quillet and Marie-Laure Pilet-Nayel for helpful discussions concerning this work, Grégoire Aubert for providing $P$. sativum RNA-seq data, Cécile Godé for assistance in PCR product sequencing and Frédéric Depta for plant care in the greenhouse. They acknowledge Eric Hanocq for providing the initial plant material for the construction of the large $P$. sativum segregating population. They would also like to thank Pascal Marget and Jean-Bernard Magnin-Robert of GRC of grain legumes, INRA, Dijon, France; Stéphane Fourtier of GRC of forage and turf species, INRA, Lusignan, France; Frederic Ottosson of NordGen, Sweden and Dave Stout of WRPIS, Pullman, Washington, USA for supplying legume seeds. They also thank the GENTYANE platform (INRA UBP, UMR 1095, Clermont-Ferrand) for genotyping. This work was partly financially supported by the Région Nord Pas-de-Calais, France (FEDER ARCIR PLANTEQ3 program). N. Tayeh was the recipient of a $\mathrm{Ph} . \mathrm{D}$. fellowship from the Ministère de l'Enseignement supérieur et de la Recherche, France.

\section{References}

Altschul SF, Gish W, Miller W, Myers EW, Lipman DJ (1990) Basic local alignment search tool. J Mol Biol 215(3):403-410. doi:10.1016/S0022-2836(05)80360-2

Arbaoui M, Link W, Satovic Z, Torres AM (2008) Quantitative trait loci of frost tolerance and physiologically related trait in faba bean (Vicia faba L.). Euphytica 164:93-104. doi:10.1007/s10681-008-9654-0

Aubert G, Morin J, Jacquin F, Loridon K, Quillet MC, Petit A, Rameau C, Lejeune-Hénaut I, Huguet T, Burstin J (2006) Functional mapping in pea, as an aid to the candidate gene selection and for investigating synteny with the model legume Medicago truncatula. Theor Appl Genet 112: 1024-1041. doi:10.1007/s00122-005-0205-y 
Bélanger G, Castonguay Y, Bertrand A, Dhont C, Rochette P, Couture L, Drapeau R, Mongrain D, Chalifour FP, Michaud R (2006) Winter damage to perennial forage crops in eastern Canada: causes, mitigation, and prediction. Can J Plant Sci 86:33-47

Bordat A, Savois V, Nicolas M, Salse J, Chauveau A, Bourgeois M, Potier J, Houtin H, Rond C, Murat F, Marget P, Aubert G, Burstin J (2011) Translational genomics in legumes allowed placing in silico 5460 unigenes on the pea functional map and identified candidate genes in Pisum sativum L. Genes Genomes Genet 1:93-103. doi:10.1534/g3.111. 000349

Brouwer DJ, Duke SH, Osborn TC (2000) Mapping genetic factors associated with winter hardiness, fall growth, and freezing injury in autotetraploid alfalfa. Crop Sci 40:1387-1396

Cannon SB, Sterck L, Rombauts S, Sato S, Cheung F, Gouzy J, Wang X, Mudge J, Vasdewani J, Schiex T, Spannagl M, Monaghan E, Nicholson C, Humphray SJ, Schoof $\mathrm{H}$ et al (2006) Legume genome evolution viewed through the Medicago truncatula and Lotus japonicus genomes. Proc Natl Acad Sci USA 103(40):14959-14964. doi:10.1073/ pnas.0603228103

Casasoli M, Derory J, Morera-Dutrey C, Brendel O, Porth I, Guehl JM, Villani F, Kremer A (2006) Comparison of quantitative trait loci for adaptive traits between oak and chestnut based on an expressed sequence tag consensus map. Genetics 172:533-546. doi:10.1534/genetics.105.048439

Castonguay Y, Michaud R, Nadeau P, Bertrand A (2009) An indoor screening method for improvement of freezing tolerance in alfalfa. Crop Sci 49:809-818. doi:10.2135/ cropsci2008.09.0539

Castonguay Y, Cloutier J, Bertrand A, Michaud R, Laberge S (2010) SRAP polymorphisms associated with superior freezing tolerance in alfalfa (Medicago sativa spp. sativa). Theor Appl Genet 120:1611-1619. doi:10.1007/s00122010-1280-2

Chen H, Wang S, Xing Y, Xu C, Hayes PM, Zhang Q (2003) Comparative analyses of genomic locations and race specificities of loci for quantitative resistance to Pyricularia grisea in rice and barley. Proc Natl Acad Sci USA 100(5):2544-2549. doi:10.1073/pnas.0437898100

Chen C, Miller P, Muehlbauer F, Neill K, Wichman D, McPhee $\mathrm{K}$ (2006) Winter pea and lentil response to seeding date and micro- and macro-environments. Agron J 98:1655-1663. doi:10.2134/agronj2006.0085

Choi HK, Mun JH, Kim DJ, Zhu H, Baek JM, Mudge J, Roe B, Ellis N, Doyle J, Kiss GB, Young ND, Cook DR (2004a) Estimating genome conservation between crop and model legume species. Proc Natl Acad Sci USA 101(43): 15289-15294. doi:10.1073/pnas.0402251101

Choi HK, Kim D, Uhm T, Limpens E, Lim H, Mun JH, Kalo P, Penmetsa RV, Seres A, Kulikova O, Roe BA, Bisseling T, Kiss GB, Cook DR (2004b) A sequence-based genetic map of Medicago truncatula and comparison of marker colinearity with $M$. sativa. Genetics 166:1463-1502

Cook DR (1999) Medicago truncatula —a model in the making! Curr Opin Plant Biol 2:301-304

Corpet F (1988) Multiple sequence alignment with hierarchical clustering. Nucl Acids Res 16(22):10881-10890
Cruz-Izquierdo S, Avila CM, Satovic Z, Palomino C, Gutierrez N, Ellwood SR, Phan HTT, Cubero JI, Torres AM (2012) Comparative genomics to bridge Vicia faba with model and closely-related legume species: stability of QTLs for flowering and yield-related traits. Theor Appl Genet 125(8):1767-1782. doi:10.1007/s00122-012-1952-1

D'Erfurth I, Le Signor C, Aubert G, Sanchez M, Vernoud V, Darchy B, Lherminier J, Bourion V, Bouteiller N, Bendahmane A, Buitink J, Prosperi JM, Thompson R, Burstin J, Gallardo K (2012) A role for an endosperm-localized subtilase in the control of seed size in legumes. New Phytol 196:738-751. doi:10.1111/j.1469-8137.2012.04296.x

Dereeper A, Guignon V, Blanc G, Audic S, Buffet S, Chevenet F, Dufayard JF, Guindon S, Lefort V, Lescot M, Claverie JM, Gascuel O (2008) Phylogeny.fr: robust phylogenetic analysis for the non-specialist. Nucl Acids Res 36:W465W469. doi:10.1093/nar/gkn180

Distelfeld A, Korol A, Dubcovsky J, Uauy C, Blake T, Fahima T (2008) Colinearity between the barley grain protein content (GPC) QTL on chromosome arm 6HS and the wheat Gpc$B 1$ region. Mol Breed 22:25-38. doi:10.1007/s11032-0079153-3

Doyle JJ, Luckow MA (2003) The rest of the iceberg. Legume diversity and evolution in a phylogenetic context. Plant Physiol 131:900-910. doi:10.1104/pp.102.018150

Dumont E, Fontaine V, Vuylsteker C, Sellier H, Bodèle S, Voedts N, Devaux R, Frise M, Avia K, Hilbert JL, Bahrman N, Hanocq E, Lejeune-Hénaut I, Delbreil B (2009) Association of sugar content QTL and PQL with physiological traits relevant to frost damage resistance in pea under field and controlled conditions. Theor Appl Genet 118:1561-1571. doi:10.1007/s00122-009-1004-7

Fatokun CA, Menancio-Hautea DI, Danesh D, Young ND (1992) Evidence for orthologous seed weight genes in cowpea and mung bean based on RFLP mapping. Genetics 132:841-846

Firıncıoğlu HK, Erbektaş E, Doğruyol L, Mutlu Z, Ünal S, Karakurt E (2009) Phenotypic variation of autumn and spring-sown vetch (Vicia sativa ssp.) populations in central Turkey. Span. J Agric Res 7(3):596-606

Gepts P, Beavis WD, Brummer EC, Shoemaker RC, Stalker HT, Weeden NF, Young ND (2005) Legumes as a model plant family. Genomics for food and feed report of the crosslegume advances through genomics conference. Plant Physiol 137:1228-1235. doi:10.1104/pp.105.060871

Gondo T, Sato S, Okumura K, Tabata S, Akashi R, Isobe S (2007) Quantitative trait locus analysis of multiple agronomic traits in the model legume Lotus japonicus. Genome 50:627-637. doi:10.1139/G07-040

Guy C (1990) Cold acclimation and freezing stress tolerance: role of protein metabolism. Annu Rev Plant Physiol Plant Mol Biol 41:187-223

Joseph B, Schlueter JA, Du J, Graham MA, Ma J, Shoemaker RC (2009) Retrotransposons within syntenic regions between soybean and Medicago truncatula and their contribution to local genome evolution. Plant Gen 2(3): 211-223. doi:10.3835/plantgenome2009.01.0001

Julier B, Flajoulot S, Barre P, Cardinet G, Santoni S, Huguet T, Huyghe C (2003) Construction of two genetic linkage maps in cultivated tetraploid alfalfa (Medicago sativa) 
using microsatellite and AFLP markers. BMC Plant Biol $3: 9$

Kahraman A, Kusmenoglu I, Aydin N, Aydogan A, Erskine W, Muehlbauer FJ (2004) QTL mapping of winter hardiness genes in lentil. Crop Sci 44:13-22

Klimenko I, Razgulayeva N, Gau M, Okumura K, Nakaya A, Tabata S, Kozlov NN, Isobe S (2010) Mapping candidate QTLs related to plant persistency in red clover. Theor Appl Genet 120:1253-1263. doi:10.1007/s00122-009-1253-5

Komlan A, Pilet-Nayel ML, Bahrman N, Baranger A, Delbreil B, Fontaine V, Hamon C, Hanocq E, Niarquin M, Sellier H,Vuylsteker C, Prosperi JM, Lejeune-Hénaut I (2013) Genetic variability and QTL mapping of freezing tolerance and related traits in Medicago truncatula. Theor Appl Genet (submitted)

Kosambi DD (1944) The estimation of map distances from recombination values. Ann Eugen 12:172-175

Kulikova O, Gualtieri G, Geurts R, Kim DJ, Cook D, Huguet T, de Jong JH, Fransz PF, Bisseling T (2001) Integration of the FISH pachytene and genetic maps of Medicago truncatula. Plant J 27:49-58

Lejeune-Hénaut I, Hanocq E, Béthencourt L, Fontaine V, Delbreil B, Morin J, Petit A, Devaux R, Boilleau M, Stempniak JJ, Thomas M, Lainé AL, Foucher F, Baranger A, Burstin J, Rameau C, Giauffret C (2008) The flowering locus $\mathrm{Hr}$ colocalizes with a major QTL affecting winter frost tolerance in Pisum sativum L. Theor Appl Genet 116:1105-1116. doi:10.1007/s00122-008-0739-x

Loridon K, McPhee K, Morin J, Dubreuil P, Pilet-Nayel ML, Aubert G, Rameau C, Baranger A, Coyne C, LejeuneHénaut L, Burstin J (2005) Microsatellite marker polymorphism and mapping in pea (Pisum sativum L.). Theor Appl Genet 111:1022-1031. doi:10.1007/s00122-0050014-3

Maughan PJ, Maroof MAS, Buss GR (1996) Molecular-marker analysis of seed-weight: genomic locations, gene action, and evidence for orthologous evolution among three legume species. Theor Appl Genet 93:574-579

Mudge J, Cannon SB, Kalo P, Oldroyd GE, Roe BA, Town CD, Young ND (2005) Highly syntenic regions in the genomes of soybean, Medicago truncatula, and Arabidopsis thaliana. BMC Plant Biol 5:15. doi:10.1186/1471-2229-5-15

Rozen S, Skaletsky HJ (2000) Primer3 on the WWW for general users and for biologist programmers. In: Krawetz S, Misener $S$ (eds) Bioinformatics methods and protocols: methods in molecular biology. Humana Press, Totowa, pp 365-386

Sadeghipour O, Aghaei P (2012) Comparison of autumn and spring sowing on performance of chickpea (Cicer arietinum L.) varieties. Int J Biosci 2(6):49-58

Sato S, Nakamura Y, Kaneko T, Asamizu E, Kato T, Nakao M, Sasamoto S, Watanabe A, Ono A, Kawashima K, Fujishiro T, Katoh M, Kohara M, Kishida Y, Minami C et al (2008) Genome structure of the legume, Lotus japonicus. DNA Res 15:227-239. doi:10.1093/dnares/dsn008
Schlueter JA, Scheffler BE, Jackson S, Shoemaker RC (2008) Fractionation of synteny in a genomic region containing tandemly duplicated genes across Glycine max, Medicago truncatula, and Arabidopsis thaliana. J Hered 99(4):390-395. doi:10.1093/jhered/esn010

Schmutz J, Cannon SB, Schlueter J, Ma J, Mitros T, Nelson W, Hyten DL, Song Q, Thelen JJ, Cheng J, Xu D, Hellsten U, May GD, Yu Y, Sakurai T et al (2010) Genome sequence of the palaeopolyploid soybean. Nature 463:178-183. doi:10.1038/nature08670

Shin JH, Van K, Kim DH, Kim KD, Jang YE, Choi BS, Kim MY, Lee SH (2008) The lipoxygenase gene family: a genomic fossil of shared polyploidy between Glycine max and Medicago truncatula. BMC Plant Biol 8:133. doi:10.1186/1471-2229-8-133

Timko MP, Rushton PJ, Laudeman TW, Bokowiec MT, Chipumuro E, Cheung F, Town CD, Chen X (2008) Sequencing and analysis of the gene-rich space of cowpea. BMC Genomics 9:103. doi:10.1186/1471-2164-9-103

Timmerman-Vaughan GM, McCallum JA, Frew TJ, Weeden NF, Russell AC (1996) Linkage mapping of quantitative trait loci controlling seed weight in pea (Pisum sativum L.). Theor Appl Genet 93:431-439

Van Ooijen JW (2006) Joinmap ${ }^{\circledR} 4$, software for the calculation of genetic linkage maps in experimental populations. Kyazma B.V, Wageningen

Van Ooijen JW (2009) MapQTL ${ }^{\circledR}$ 6, software for the mapping of quantitative trait loci in experimental populations of diploid species. Kyazma B.V, Wageningen

Varshney RK, Chen W, Li Y, Bharti AK, Saxena RK, Schlueter JA, Donoghue MTA, Azam S, Fan G, Whaley AM, Farmer AD, Sheridan J, Iwata A, Tuteja R, Penmetsa RV et al (2012) Draft genome sequence of pigeonpea (Cajanus cajan), an orphan legume crop of resource-poor farmers. Nat Biotechnol 30(1):83-89. doi:10.1038/nbt.2022

Voorrips RE (2002) MapChart: software for the graphical presentation of linkage maps and QTLs. J Hered 93(1):77-78

Young ND, Debellé F, Oldroyd GED, Geurts R, Cannon SB, Udvardi MK, Benedito VA, Mayer KFX, Gouzy J, Schoof H, Van De Peer Y, Proost S, Cook DR, Meyers BC, Spannagl M et al (2011) The Medicago genome provides insight into the evolution of rhizobial symbioses. Nature 480:520-524. doi:10.1038/nature10625

Zhu H, Cannon SB, Young ND, Cook DR (2002) Phylogeny and genomic organization of the TIR and Non-TIR NBS-LRR resistance gene family in Medicago truncatula. Mol Plant Microbe Interact 15(6):529-539

Zorrilla-Fontanesi Y, Cabeza A, Domínguez P, Medina JJ, Valpuesta V, Denoyes-Rothan B, Sánchez-Sevilla JF, Amaya I (2011) Quantitative trait loci and underlying candidate genes controlling agronomical and fruit quality traits in octoploid strawberry (Fragaria $\mathrm{x}$ ananassa). Theor Appl Genet 123:755-778. doi:10.1007/s00122-011-1624-6 